\title{
Estudio neuropsicológico basado en la creatividad, las inteligencias múltiples y la función ejecutiva en el ámbito educativo
}

\author{
Marta Castillo Delgado, Armando Ezquerro Cordón, Fátima Llamas Salguero y Verónica López \\ Fernández. Universidad Internacional de la Rioja
}

Recepción: 18 de enero de 2016 | Revisión: 19 de enero de 2016 | Aceptado: 21 enero de 2016

Correspondencia: veronica.lopez@unir.net

Citar: Castillo-Delgado, M., Ezquerro-Cordón, A., Llamas-Salguero, F. y López-Fernández, V. (2016). Estudio neuropsicológico

basado en la creatividad, las inteligencias múltiples y la función ejecutiva en el ámbito educativo. ReiDoCrea, 5, 9-15.

\begin{abstract}
Resumen: Durante los últimos años la neuropsicología supone un enfoque de gran interés en su aplicación en el ámbito educativo. Por ello, es fundamental estudiar la relación que existe entre diferentes funciones cognitivas, en aras de optimizar el aprendizaje del alumnado. Método: La investigación se realizó en un colegio público de Sevilla, con participantes $(n=29)$ de edades comprendidas entre 8 y 10 años, escolarizados en el cuarto curso de Educación Primaria. Se les aplicaron diferentes pruebas para evaluar las inteligencias múltiples, la creatividad y las funciones ejecutivas. Posteriormente se analizó la relación entre las variables estudiadas. Resultados: El análisis estadístico muestra que existe relación significativa entre creatividad gráfica e inteligencia interpersonal, entre creatividad verbal e inteligencia lingüística, y entre creatividad total e inteligencia interpersonal; Sin embargo no se encontraron valores significativos entre las variables de creatividad y función ejecutiva. Conclusiones: Es necesario realizar más investigaciones para esclarecer las posibles relaciones existentes entre Creatividad e Inteligencias Múltiples.

Palabras clave: Método Educativo | Proceso Cognitivo
\end{abstract}

Neuropsychological Study based on Creativity, Multiple Intelligences and Executive Function in Education

\begin{abstract}
Neuropsychology is a focus of great interest in their application in education. Therefore, it is essential to study the relationship between different cognitive functions in order to optimize student learning. Method: The research was conducted in a Public School in Seville, with participants $(n=29)$ aged, between 8 and 10 years, they are enrolled in the fourth year of Primary Education. Different tests were applied to evaluate multiple intelligences, creativity and executive functions. Then the relationship between the variables studied was analyzed. Results: Statistical analysis shows that there is significant relationship between graphic creativity and interpersonal intelligence, including verbal linguistic intelligence and creativity, and between full creativity and interpersonal intelligence; However, no significant values among the variables of creativity and executive function were found. Conclusions: It is necessary to do more research to clarify the possible relationship between Creativity and Multiple Intelligences.
\end{abstract}

Keywords: Educative Method | Cognitive Process

Aunque la escuela debiera ser permeable a las exigencias de la sociedad actual y a las necesidades de las personas que la forman, tal y como afirman Bordieu y Passeron (1979) la es-cuela persiste en su afán "reproductivo", de transmisión y conservación de los valores de una sociedad. Este afán es el germen de lo que Amabile (2011) llamó "dilema educativo". Este término describe la tensión entre la enseñanza de hábitos cognitivos y la de hábitos creativos. Sternberg (1997) también denuncia que en las escuelas se favorece más el pensamiento inerte que el pensamiento creativo.

En contrapartida al carácter estático de la escuela hacia la que apuntan las afirmaciones de los autores anteriores, se encuentra la tesitura de las posibilidades que por definición tendría el constructo de creatividad para formar a individuos competentes para la vida en sociedad. Como defiende la UNESCO, la creatividad es un elemento esencial no sólo de la vida espiritual, sino también de la vida material y económica de individuos y pueblos. Tal y como señalan Marina y Marina (2013) cuando estamos hablando de creatividad no estamos hablando de actividades artísticas, sino de una manera de enfrentarse con la vida, sus oportunidades y sus problemas.

Considerando la creatividad como una oportunidad para formar personas capaces de adaptarse a los cambios y de crear soluciones y retos, estamos ante una pregunta clave: ¿es posible "enseñar" creatividad? Al respecto, Vygotsky (1981) consideraba 
que la creatividad existe potencialmente en todos los seres humanos, y es susceptible de desarrollar, o sea, que no es privativa de los genios, sino que está presente en cualquier ser humano que imagine, transforme o cree algo por insignificante que sea en comparación con las grandes personalidades creativas de la historia. Este planteamiento que ha sido ratificado en numerosas investigaciones contemporáneas ha dado lugar a que la enseñanza de la creatividad se materialice en proyectos, programaciones, se incorpore a todas las materias curriculares y se traduzca a actividades concretas (Marín y De La Torre, 1991) .

Además de la relevancia e implicación de la creatividad en el aula, se torna de interés la inclusión de la teoría de las inteligencias múltiples para atender a la diversidad en la escuela, junto con otra variables neuropsicológica es la concepción de las funciones ejecutivas, por su importancia para el proceso de enseñanza-aprendizaje (TirapuUstárroz, Muñoz-Céspedes y Pelegrín-Valero, 2002).

Según Gardner (2003, 1993), con respecto al concepto de inteligencias múltiples, "se trata de una visión pluralista de la mente, que reconoce muchas facetas distintas de la cognición, que tiene en cuenta que las personas tienen diferentes potenciales cognitivos y que contrasta diferentes estilos cognitivos". Esta visión diversificada de la inteligencia aplicada a los procesos de enseñanza-aprendizaje que se practican en las escuelas arrojaría una oportunidad real de atender a la gran diversidad cognitiva, personal, cultural, económica, social, etc. que existen actualmente en las escuelas respetando cánones de inclusión. La sobrevaloración y primacía de las áreas curriculares instrumentales relegan a un segundo plano al alumnado que podría tener experiencias educativas exitosas si se contemplaran actividades que ofertaran oportunidades de aplicar las múltiples inteligencias. También Gardner y Gardner (2012) afirman que hay cinco mentes las cuales son: la mente disciplinada, la mente sintética, la mente creativa, la mente respetuosa y la mente ética.

En base a lo comentado, el objetivo de este trabajo es analizar la relación entre las variables del estudio, esto es, conocer si existe relación estadísticamente significativa entre la creatividad y cada una de las inteligencias múltiples de la muestra; ratificar de forma empírica la relación entre los procesos cognitivos implicados en tareas de creatividad y las funciones ejecutivas, así como dilucidar si existe relación entre las inteligencias múltiples y las funciones ejecutivas.

\section{Método}

\section{Participantes}

La muestra que participó en la investigación corresponde a un grupo de 29 estudiantes de $4^{\circ}$ curso de Educación Primaria de un Colegio Público situado en el barrio sevillano Polígono San Pablo. El alumnado que atiende el centro proviene, fundamentalmente del propio barrio. El porcentaje de personas inactivas en el área de influencia supera el $60 \%$. El nivel educativo de la población se encuentra en términos generales por debajo de la media de Sevilla estando el nivel de estudios universitarios en un $4,61 \%$. En cuanto a la población de extranjeros censados se ha incrementado en un $25 \%$. La nacionalidad de la población extranjera de la zona, en cuanto a los principales países de procedencia, es Ecuador, Marruecos, Senegal, Colombia, Bolivia y Perú. En cuanto al nivel educativo de la población extranjera destaca el elevado porcentaje que tiene estudios medios o superiores, un 51\%, junto con la práctica inexistencia de analfabetismo. Debido al alto nivel de envejecimiento de la población y las estructuras monoparentales, es de destacar el alto índice de alumnado que depende en su seguimiento educativo y personal de las abuelas. La población de inmigrantes ha 
aumentado aunque está situada aún en porcentajes menores que en el resto de la ciudad y siendo mayoritario el origen castellano parlante no suponen problemas añadidos de adaptación. Es de destacar, casi en la generalidad, una diferencia positiva en cuanto a hábitos de relaciones sociales y comunicativas. La muestra intencional de la investigación se corresponde con un grupo de 29 alumnos/as de $4^{\circ}$ curso de Educación Primaria formado por 20 niñas y 9 niños. Dentro del grupo de alumnos/as, 2 de ellos siguen un programa de adaptación curricular significativa y reciben refuerzo educativo junto con otros tres compañeros/as. (Ver tabla 1)

\begin{tabular}{|ccc|}
\hline \multicolumn{3}{|c|}{ Tabla 1. Descripción detallada de la muestra por sexo y edad } \\
\hline Edad en años & Niñas & Niños \\
\hline 8 & 9 & 3 \\
9 & 11 & 4 \\
10 & 0 & 2 \\
\hline
\end{tabular}

\section{Instrumentos}

Para medir la variable inteligencias múltiples se utilizó el Cuestionario de Inteligencias Múltiples de Armstrong. Este cuestionario consta de ocho apartados que corresponden a cada una de las Inteligencias Múltiples que son Lingüística, Matemática, Espacial, Naturalista, Corporal-Cenestésica, Musical, Interpersonal e Intrapersonal. Cada uno de estos apartados tienen 10 ítems, que se evalúan con SI vale 1 punto, NO computa como 0 puntos y A VECES vale 0'5 puntos. Luego con esta puntuación se puede sacar el índice de inteligencia en cada uno de los apartados. Evaluados con: de 0 a 2 nivel bajo, de $2^{\prime} 5$ a 4 nivel medio-bajo, de $3{ }^{\prime} 5$ a 6 nivel medio, de $6{ }^{\prime} 5$ a 8 nivel medioalto y de 8'5 a 10 nivel alto.

Para medir la variable de creatividad de la muestra se ha utilizado el Test de diagnóstico de Creatividad de Marín. La prueba es una simplificación del Test de Creatividad de Torrance y puede aplicarse a partir de los seis años y requiere que los sujetos dominen la escritura. El test de Marín tiene dos partes diferenciadas que miden la creatividad verbal y la creatividad gráfica. La prueba verbal presenta una imagen y una hoja con cuarenta líneas numeradas en las que escribir las innumerables cuestiones que pudiera suscitar la imagen. La prueba gráfica consta de dos ejercicios. El primero de ellos presenta un conjunto de figuras organizadas en siete filas en las que se repite el mismo dibujo cinco veces. Las tres primeras filas están formadas por figuras cerradas y las siguientes tres filas aparecen figuras abiertas. En la última fila se presentan estímulos más complejos formados por dos segmentos independientes. En el segundo ejercicio se presentan también treinta y cinco figuras pero todas las figuras son diferentes y están enmarcadas en cuadrados. Las dos primeras filas están compuestas por figuras cerradas y las cinco filas restantes por líneas sueltas. Al comienzo de la aplicación, conviene dar unas orientaciones básicas acerca de la realización de la prueba como dibujar/escribir el máximo número de respuestas, intentar que las respuestas sean lo más diferentes posible entre ellas y con respecto a las que puedan realizar los demás compañeros/as. El test de Marín pretende medir fundamentalmente las variables productividad, flexibilidad mental, originalidad y la elaboración.

Para medir la variable función ejecutiva se ha utilizado la tarea "Simón dice" adaptada de la prueba elaborada originalmente de La Voie. La versión original está estructurada en cuatro partes pero en esta investigación fue diseñada una versión abreviada de dos partes. En cualquier caso, esta tarea se caracteriza por presentar un estímulo verbal que requerirá una respuesta motriz o la inhibición de esta. Para la aplicación de la prueba se requiere la participación de dos adultos que dan el estímulo verbal a la clase, observan y registran las respuestas de forma alterna. La primera parte de la 
prueba consiste en diez instrucciones dadas al alumnado bajo la con-signa de que deben realizar la orden que escuchan solo si va precedida de "Simón dice" -por ejemplo: "Simón dice aplaudir"- e inhibirla en los casos en los que la orden no vaya precedida de "Simón dice" -por ejemplo: "sentarse"-. Los adultos que aplican la prueba no realizan ninguna de las actividades. En la segunda parte de la prueba, las órdenes que los/as niños/as deben inhibir van precedidas por el término "no" -por ejemplo: "no sentarse"-. En esta parte de la prueba los adultos realizan las instrucciones, añadiendo así un estímulo de interferencia. La valoración de la prueba atiende a cuatro ítems: error valorado con 0 puntos, tardanza -lapso de dos segundos o más- y corrección valorados con 1 punto y dos puntos para el acierto.

\section{Procedimiento}

El primer paso que se realizó fue pedir permiso al equipo directivo del centro para poder aplicar las pruebas. Una vez dado el visto bueno, se seleccionó el grupo más numeroso del centro para que la muestra fuera significativa. Se le explicó a la tutora del curso en cuestión, el objetivo del estudio, las pruebas que eran necesarias realizar y el tiempo que tomaría realizarlas. El cuestionario de inteligencias múltiples fue realizado por las familias a las que se les facilitó el test a través de sus hijos/as junto con una nota pidiendo su colaboración. Las pruebas para evaluar la creatividad y la función ejecutiva fueron realizadas en un periodo de una hora en el colegio. La primera de las pruebas que realizaron los alumnos/as fue la de Creatividad para lo cual contaron con una breve explicación y ejemplos de cómo resolverlas. El tiempo que tuvieron para realizar la prueba fue de 30 minutos. La prueba para valorar la función ejecutiva se realizó de forma grupal a través de la actividad "Simón dice" y con la colaboración de la tutora para la evaluación de la misma.

\section{Análisis de datos}

El análisis de los datos se realiza a través de los programas Excel de Microsoft y el programa estadístico SPSS versión 20 . Se realiza un análisis descriptivo y un análisis de correlación de Pearson.

\section{Resultados}

Los resultados descriptivos obtenidos, como puede verse en la Tabla 2, la cual muestra los resultados de las variables de Creatividad Gráfica, Verbal y Total. Los resultados muestran un porcentaje más elevado de Creatividad Gráfica en relación a la Creatividad Verbal). Aún así, tanto la creatividad verbal, la gráfica como la total, muestran un desempeño bajo en el conjunto de la muestra evaluada.

\begin{tabular}{|lcc|}
\hline \multicolumn{3}{|c|}{ Tabla 2. Resultados descriptivos obtenidos en la variable Creatividad } \\
\hline & Media & Desviación típica \\
\cline { 2 - 3 } Creatividad Grafica & 36,86 & 7,18 \\
Creatividad Verbal & 21,06 & 3,41 \\
Creatividad Total & 57,13 & 9,43 \\
\hline
\end{tabular}

A continuación se muestran los datos relativos a los diferentes tipos de inteligencias múltiples (Tabla 3). Tal y como puede verse en la tabla, la inteligencia que muestra mayor desarrollo en la muestra es la inteligencia lingüística, seguida de la interpersonal. Sin embargo, la que menos desarrollada se muestra es la matemática. Estos resultados muestran que la distribución de las puntuaciones en cada inteligencia son diferentes en la muestra, exhibiendo puntos fuertes y débiles. 


\begin{tabular}{|c|c|c|}
\hline Tipo de Inteligencia Múltiple & Media & Desviación típica \\
\hline Lingüística & 7,64 & 1,37 \\
\hline Matemática & 6,52 & 1,80 \\
\hline Musical & 7,14 & 1,18 \\
\hline Naturalista & 6,98 & 1,44 \\
\hline Corporal-Kinestésica & 6,91 & 1,21 \\
\hline Viso-Espacial & 6,64 & 1,44 \\
\hline Interpersonal & 7,59 & 0,85 \\
\hline Intrapersonal & 7,05 & 1,56 \\
\hline
\end{tabular}

Por último, se muestran la media y desviación típica de la variable función ejecutiva (Tabla 4). Como puede verse, la media obtenida empleando la prueba de "Simón dice" es de 19,72 aciertos. Teniendo en cuenta que la puntuación máxima posible es de 20 puntos, la puntuación obtenida es alta.

\begin{tabular}{|ccc|}
\hline \multicolumn{3}{|c|}{ Tabla 4. Resultados descriptivos obtenidos en Funciones Ejecutivas } \\
\hline \multirow{3}{*}{ Función Ejecutiva } & Media & Desviación típica \\
\cline { 2 - 3 } & 19,72 & 0,59 \\
\hline
\end{tabular}

Posteriormente se realizó un análisis de la correlación de Pearson. En la Tabla 5 se aprecian los resultados obtenidos entre Creatividad Gráfica e Inteligencias Múltiples. Los resultados muestran que sólo existe correlación estadísticamente significativa y directa entre los valores de Creatividad e Inteligencia Interpersonal, que es igual a 0,449 y tiene una probabilidad asociada $p=.015$. Por lo tanto, se puede afirmar la existencia de correlación entre estás variables. Esta correlación es directa y de intensidad media, la cual se denomina emocional.

\begin{tabular}{|c|c|c|c|c|c|c|c|c|c|c|}
\hline \multicolumn{11}{|c|}{ Tabla 5. Correlación de Pearson entre Creatividad Gráfica e Inteligencias Múltiples } \\
\hline Creativid & & Lingüística & Matemática & Musical & Naturalista & Kinestésica & Espacial & Interpersonal & Intrapersonal & Ejecutiva \\
\hline \multirow[t]{2}{*}{ Grafica } & C. Pearson &, 247 & ,145 &,- 042 & ,263 &,- 012 &, 005 &, $449^{*}$ &, 136 &,- 127 \\
\hline & Sig. (bilateral) & ,196 & ,452 & ,829 & ,168 & ,952 & ,978 & ,015 & ,482 &, 512 \\
\hline
\end{tabular}

En cuanto a la Creatividad Total y las Inteligencias Múltiples, tal como se puede ver en la Tabla 6 , sólo se ha encontrado correlación estadísticamente significativa entre la Creatividad Total y la Inteligencia Interpersonal. La correlación entre Creatividad Total e Inteligencia Interpersonal es igual a 0,406 y tiene una probabilidad asociada $p=.029$. Es decir, se puede afirmar que es significativa. En cambio, en el resto de las inteligencias no se ha encontrado relación significativa.

\begin{tabular}{|lccccccccccc|}
\hline \multicolumn{10}{c|}{ Tabla 6. Correlación de Pearson entre Creatividad Total e Inteligencias Múltiples } \\
\hline $\begin{array}{l}\text { Creatividad } \\
\text { Total }\end{array}$ & Lingüística & Matemática & Musical & Naturalista & Kinestésica & Espacial & Interpersonal & Intrapersonal & Ejecutiva \\
\cline { 2 - 11 } & Pearson &, 242 &, 114 &, 032 &, 227 &, 060 &, 218 & $\mathbf{4 0 6}$ &, 116 &,- 191 \\
\cline { 2 - 11 } & Sig.(bilateral) &, 206 &, 554 &, 869 &, 236 &, 755 &, 255 &, 029 &, 549 &, 320 \\
\hline
\end{tabular}

Respecto a la correlación entre Creatividad Verbal e Inteligencias, puede verse que sólo es significativa la correlación entre la primera y la Lingüística, siendo igual a 0,495 y tiene una probabilidad asociada (sig. bilateral) igual a 0,006. En cambio, en el resto de las inteligencias o la función ejecutiva no se han encontrado ninguna relación significativa con la Creatividad Verbal.

Tabla 7. Correlación de Pearson entre Creatividad Verbal, Función Ejecutiva e Inteligencias Múltiples

\begin{tabular}{|c|c|c|c|c|c|c|c|c|c|c|}
\hline \multirow{3}{*}{$\begin{array}{l}\text { Creativid } \\
\text { Verbal }\end{array}$} & & Lingüística & Matemática & Musical & Naturalista & Kinestésica & Espacial & Interpersonal & Intrapersonal & Ejecutiva \\
\hline & C. Pearson &, $495^{* *}$ & ,218 &, 100 & ,146 &,- 094 &, 333 & 134 & 311 &,- 150 \\
\hline & Sig. (bilateral) & ,006 & ,256 & 607 & ,449 & ,629 & ,078 & ,488 & ,100 &, 439 \\
\hline
\end{tabular}


Por último, en relación a la correlación entre las Inteligencias Múltiples y la Función Ejecutiva, no se encontró relación estadísticamente significativa después de realizar el análisis estadístico, tal como se puede apreciar en la tabla 8.

\begin{tabular}{|c|c|c|c|c|c|c|c|c|c|}
\hline \multicolumn{10}{|c|}{ Tabla 8. Correlación de Pearson entre Función Ejecutiva e Inteligencias Múltiples } \\
\hline & & Lingüística & Matemática & Musical & Naturalista & Kinestésica & Espacial & Interpersonal & Intrapersonal \\
\hline Función & C. Pearson &,- 040 & ,172 & ,185 &,- 131 & ,090 &,- 038 & ,014 & $\begin{array}{l}-119 \\
\end{array}$ \\
\hline Ejecutiva & Sig. (bilateral) & 839 & ,371 & ,338 & ,499 & ,641 & ,846 & ,944 & ,537 \\
\hline
\end{tabular}

\section{Discusión}

Este estudio confirma que existe una relación significativa entre la Creatividad Gráfica y la Inteligencia Interpersonal, entre la Creatividad Verbal y la Inteligencia Lingüística, y entre la Creatividad Total y la Inteligencia Interpersonal. De los diferentes planteamientos acerca de la relación entre creatividad e inteligencia revisados en el marco teórico, los resultados obtenidos en este estudio se encuadran en la vertiente que aceptan la hipótesis de que inteligencia y creatividad son dos constructos diferentes, pero se existe una fuerte relación entre ambos. Por lo tanto los resultados son coincidente con la línea de investigación de Renzulli (1977), quien propone la teoría de los tres anillos -creatividad, inteligencia y persistencia en la tarea- y a través de esta se establece que creatividad e inteligencia son realidades distintas, pero en algunas ocasiones se superponen.

En cuanto a la correlación entre las variables de Creatividad y Función Ejecutiva, los resultados obtenidos no evidencian valores significativos de correlación entre ambas variables; por lo tanto, no se obtienen datos que permitan relacionarla de manera lineal (Arieti, 1976; Martindale y Daily, 1996; Carlsson (2002).

Por último, respecto a la correlación entre las Inteligencias Múltiples y la Función Ejecutiva. Los resultados obtenidos muestran que no existe correlación significativa. Por lo tanto, no se verifica el estudio realizado por Artigas-Pallarés, Rigau-Ratera y García-Nonell (2007) que ponía de manifiesto la relación entre ambas variables. Tampoco se puede establecer ninguna correlación entre ambas variables que permita discutir la idea de la Inteligencia Ejecutiva entendida como motor de activación del resto de las inteligencias.

Para concluir, queda mucho por investigar respecto a este área, para esclarecer la relación existente entre las variables estudiadas a través de estudio de investigación exploratoria. Por lo tanto, es necesario realizar más investigaciones para aclarar la posible relación entre Creatividad e Inteligencias Múltiples.

Referencias

Amabile, T., \& Kramer, S. (2011). The Progress Principle: Using Small Wins to Ignite Joy, Engagement, and Creativity at Work. Boston: Harvard Business Review Press.

Arieti, S. (1976). Creativity: The Magic Synthesis. New York: Basic Books.

Artigas-Pallarés, J., Rigau-Ratera, E. y García-Nonell, C. (2007). Relación entre capacidad de inteligencia límite y trastornos del neurodesarrollo. Revista de Neurología, 44(12), 739-744.

Bordieu P., Passeron JC. (1979). La reproducción. Barcelona: Laia.

Carlsson, I. (2002). Anxiety and Flexibility of Defense Related to High or Low Creativity. Creativity Research Journal, 14(3-4), 341-349. 
Gardner, H. (2003). Multiple Intelligences After Twenty Years. Chicago: American Educational Research Association.

Gardner, J. (Ed.). (2012). Assessment and Learning. London: Sage.

Marín, R. y. de la Torre, S. (1991). Manual de Creatividad: Aplicaciones Educativas. Barcelona: Vicens-Vives.

Marina, J.A. y Marina, E. (2013). El aprendizaje de la creatividad. Barcelona: Ariel.

Martindale, C., \& Dailey, A. (1996). Creativity, Primary Process Cognition and Personality. Personality and Individual Differences, 20(4), 409-414.

Renzulli, J.S. (1977). The Enrichment Triad Model: A Guide for Developing Defensible Programs for the Gifted and Talented. Wethersfield: Creative Learning Press.

Sternberg, R.J. (1997). Inteligencia exitosa. Barcelona: Paidós.

Tirapu-Ustárroz, J., Muñoz-Céspedes, J.M. y Pelegrín-Valero, C. (2002). Funciones ejecutivas: necesidad de una integración conceptual. Revista de Neurología, 34(7), 673-685.

Vygotsky, LS. (1981). Pensamiento y palabra. Infancia y Aprendizaje, 4(sup1), 15-35. 